\title{
Recent Results in Neutrino Astronomy
}

\author{
C. Kopper* \\ Department of Physics, CCIS 4-181, University of Alberta, Edmonton, Alberta T6G 2E1, \\ Canada \\ E-mail: kopper@ualberta.ca
}

With the recent discovery of high-energy neutrinos of extra-terrestrial origin by the IceCube neutrino observatory, neutrino-astronomy is entering a new era. The highest energy neutrinos observed to date exceed $1 \mathrm{PeV}$ in energy, a regime of particular interest because the neutrinos should point back to the still elusive accelerators of the highest energy Galactic and extragalactic cosmic rays. This review will cover currently operating neutrino telescopes in water and ice, the latest results from searches for a flux of extra-terrestrial neutrinos and current efforts in the search for steady and transient neutrino point sources. In addition, future detectors such as KM3NeT in the Mediterranean Sea and plans for IceCube detector upgrades will be discussed.

The 34th International Cosmic Ray Conference,

30 July- 6 August, 2015

The Hague, The Netherlands

${ }^{*}$ Speaker. 


\section{Introduction}

With the recent discovery of high-energy neutrinos of extra-terrestrial origin by the IceCube neutrino observatory[1, 2], neutrino-astronomy is entering a new era. Even long before this discovery, neutrinos have been targeted as ideal cosmic messengers: they only interact weakly and can thus escape from dense astrophysical environments that are opaque to electro-magnetic radiation. They are not affected by Galactic and intergalactic magnetic fields and will thus travel in straight paths, pointing back to their sources. Measuring high-energy astrophysical neutrinos arriving at Earth provides insight into a large number of astrophysical phenomena, ranging from supernova explosions to acceleration processes in active galactic nuclei. Because they are only created in hadronic processes, they can also help to solve the 100-year old mystery of the acceleration of cosmic rays. Finding a class of Galactic and/or extra-galactic neutrino sources would make them smoking guns for the acceleration sites of cosmic rays.

This article will give a brief overview of neutrino flux expectations, of current and future detectors, the detection principle and various detection channels. It will then highlight a few important recent results and briefly touch on future detectors. A discussion of the multi-messenger connection of the high-energy astrophysical neutrino observation [3] and a full overview of highlight results in neutrino astronomy [4] can be found later in these proceedings.

\section{Neutrino Flux Expectation}

Given the abundant number of observed high-energy hadronic cosmic rays, a flux of neutrinos from interactions of these cosmic rays with ambient matter and radiation fields is expected. These interactions can either happen at a source or during propagation through space. As an example, a high-energy proton interacting with a target photon will yield charged and neutral pions in processes like $p \gamma \rightarrow n \pi^{+}$and $p \gamma \rightarrow p \pi^{0}$. While neutral pions decay into two gammas $\left(\pi^{0} \rightarrow 2 \gamma\right)$ and create a flux of high-energy gamma rays, charged pions eventually decay to neutrinos. From charged-pion decay, a flavor ratio of $v_{e}: v_{\mu}: v_{\tau}$ of $1: 2: 0$ is expected. Due to oscillations, this corresponds to a flavor ratio at Earth of approximately $1: 1: 1$ assuming a source distance much larger than the oscillation length scale. The expected ratio can change in case of different source environments such as in the presence of strong magnetic fields.

A rough estimate of the neutrino flux expected (assuming a proton-only cosmic ray injection, a purely extra-galactic origin and considering only a single interaction of protons converted into neutrons and charged pions) is provided by the so-called 'Waxman-Bahcall bound' [5]. It sets an upper bound for the all-flavor flux of neutrinos at $E^{2} \Phi \approx 3 \cdot 10^{-8} \mathrm{GeVcm}^{-2} \mathrm{~s}^{-1} \mathrm{sr}^{-1}$. Various detailed model predictions are available for a wide range of potential source classes, such as gamma-ray bursts and active galactic nuclei [6].

All backgrounds to astrophysical neutrino detection are due to interactions of cosmic ray air showers. 'Atmospheric muons', produced by $\pi$ and $K$ decays in the atmosphere above a detector can penetrate to reach the detector, even through substantial amounts of shielding material. The same interactions also produce neutrinos that enter the detector from above and below. These 'atmospheric neutrinos' are seen at a lower rate due to the lower neutrino interaction cross-section [7]. The flavor composition of the atmospheric neutrino flux is heavily biased towards muon neutrinos 
because $\pi$ and $K$ mesons decay overwhelmingly to muons rather than electrons. Because of the increasing lifetime of the parent mesons with increasing energy, their interaction probability in the atmosphere increases. This leads to a suppression at high energies producing a spectrum approximately one power steeper than the original primary cosmic ray spectrum [8]. At higher energies of about $100 \mathrm{TeV}$ a similar flux of muons and neutrinos from the decay of charmed mesons is expected to become important. Because of the shorter lifetime of these particles, no suppression from interaction before decay is expected, leading to an expected cross-over with the neutrino flux from $\pi / K$ [9]. This 'prompt' atmospheric neutrino flux has not yet been observed and is only poorly constrained at the moment.

\section{Detection Principle}

In order to detect a flux on the order of the Waxman-Bahcall bound within a few years, detectors on the scale of a cubic kilometer are necessary. Building such detectors requires large amounts of natural transparent medium, such as water or ice. The principle of detection is similar for all detectors: Neutrinos are detected by observing the Cherenkov light produced by charged particles created when neutrinos interact. These particles generally travel distances too small to be resolved individually and the particle shower (or 'cacade') is observed only in aggregate. In $v_{\mu}$ chargedcurrent (CC) interactions, however, as well as a minority of $v_{\tau} \mathrm{CC}$ interactions, a high-energy muon is produced that leaves a visible track (unless produced on the detector boundary heading outward). Although the deposited energy resolution is similar for all events, the angular resolution for events containing visible muon tracks is much better $\left(\lesssim 1^{\circ}, 50 \%\right.$ CL in ice, better in water) than for those that do not $\left(\sim 15^{\circ}, 50 \% \mathrm{CL}\right.$ in ice, better in water) [10]. For equal neutrino fluxes of all flavors (1:1:1), $v_{\mu}$ CC events make up only $20 \%$ of interactions. Tau neutrinos can have other signatures, such as "double-bang" events with two showers close to each other. Such signatures, expected to be detectable at the PeV level, have not yet been observed. Searches for double-pulse signatures from $v_{\tau}$ at lower energies are on-going [11].

Because muons at the relevant energies can travel over distances of several kilometers, one way to look for neutrinos is to search for track events entering the detector. To suppress the overwhelming number of down-going atmospheric muons $\left(\approx 10^{6}\right.$ higher than the rate expected from neutrinos) only up-going events in the detector are selected. This effectively uses the Earth to shield against the atmospheric muon background. Atmospheric neutrinos are an irreducible background in this case. They need to be included in analyses on a statistical basis and described by their energy spectrum and angular distribution. The other main way to select a reasonably pure neutrino event sample is to use the outer layers of the detector as an anti-coincidence veto. Incoming tracks from atmospheric muons will be rejected while neutrinos interacting inside the detector will be able to pass the veto. This significantly reduces the effective detection volume compared to the "track" channel described above but it allows to detect all three flavors and allows for all-sky observation. The veto will even suppress some of the down-going atmospheric neutrino flux because the veto is triggered by muons traveling in coincidence with the neutrino generated in the same cosmic ray air shower $[12,13]$. 


\section{Neutrino Detectors}

Several large-scale neutrino telescopes are currently in operation around the world. All of these use the same detection principle for Cherenkov light: they instrument a large volume of natural water or ice with a three-dimensional array of photomultiplier tubes (PMTs) contained in pressure-resistant glass housings, so-called 'optical modules'. These optical modules record the arrival time and amplitude of photons. Dedicated algorithms use a model of the optical properties of water or ice (scattering, absorption and noise) to reconstruct the event direction, topology and energy in the detector.

In the Mediterranean sea, several efforts were started at different sites: the NESTOR project off the coast of Greece [14], the NEMO project close to Sicily [15] and the ANTARES neutrino telescope off the coast of France. While the first two projects made significant contributions to current and future deep-sea neutrino detector technology, the ANTARES collaboration has been operating a large-scale detector in stable conditions for several years. The ANTARES detector consists of 12 vertical "strings" with 25 storeys of consisting of three optical modules each. The total instrumented volume of the detector, completed in 2008, is approximately $0.01 \mathrm{~km}^{3}$. ANTARES and the future Mediterranean cubic-kilometer detector KM3NeT are highlighted in a separate contribution in these prcoeedings [16].

Another neutrino detector, NT200, is deployed in Lake Baikal using the frozen lake surface during the winter as a deployment platform. This version of the detector consisting of 8 strings with 192 optical modules in total was completed in 1998. An upgrade, NT200+, adding three more strings with 12 modules each was deployed in 2005. The currently operating detector is smaller than ANTARES, but a future gigaton upgrade is planned and a first cluster of this upgrade was deployed in early 2015 [17].

Currently, the largest neutrino telescope is the IceCube neutrino telescope, located at the geographical South Pole [18]. It was deployed between 2005 and 2010 and consists of 86 strings with 5160 optical modules in total. The instrumented volume is a cubic kilometer at a depth between $1450 \mathrm{~m}$ and $2450 \mathrm{~m}$. Unlike the water-based detectors described previously it is deployed in the antarctic glacier. The detector contains a denser sub-array called DeepCore lowering the energy threshold for neutrino physics studies using the atmospheric neutrino flux as a source. It also is complemented by an air-shower array on the surface of the glacier called IceTop [19].

\section{Neutrino Searches}

\subsection{Diffuse Neutrino Flux Searches}

Before the discovery of the astrophysical neutrino flux mentioned earlier, hints of it had been seen in various analyses of data of the partially completed IceCube detector, both in the track channel [20] and in the cascade channel [21]. The discovery of two PeV energy cascades in a two-year search for GZK neutrinos (from cosmic rays interacting on photons of the cosmic microwave background) provided the first concrete evidence for an astrophysical flux. The detected event energies were too low for a GZK flux but higher than expected from atmospheric backgrounds and thus provided further evidence for an unaccounted component in the measured flux. A dedicated search for events with the neutrino vertex inside the detector using the outer layer of optical modules as 


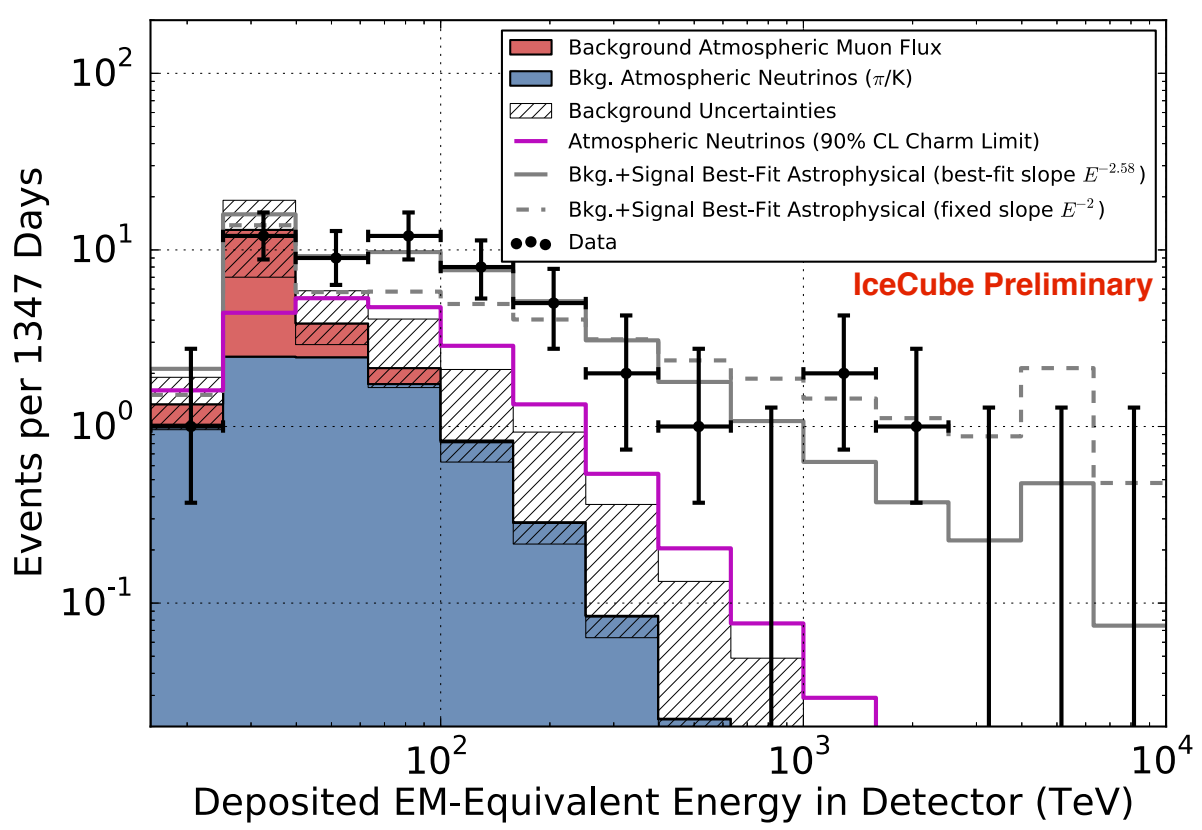

Figure 1: Deposited energies of the observed events in four years of the high-energy starting event search in IceCube. [22].

a veto (as explained above) finally provided evidence on the $>3 \sigma$ level against the atmosphericonly null-hypothesis [1]. A follow-up extending this analysis to three years of data increased the significance to $5.7 \sigma$. Continuing this analysis with a fourth year of data increases this significance further (see [22] in these proceedings). A total of 54 events are detected in the whole 4-year sample. Their energy spectrum is shown in Fig. 1. Assuming a 1:1:1 flavor ratio and a 1:1 neutrino to anti-neutrino ratio, the best fit per-neutrino flux for a sample of four years of data is

$$
E^{2} \phi(E)=2.2 \pm 0.7 \times 10^{-8}(E / 100 \mathrm{TeV})^{-0.58 \pm 0.25} \mathrm{GeVcm}^{-2} \mathrm{~s}^{-1} \mathrm{sr}^{-1}
$$

The choice of $100 \mathrm{TeV}$ as the pivot point minimizes the correlation between the spectral index and the normalization. It should also be noted that the detected flux is on the order of the WaxmanBahcall bound, although the spectral index and normalization are still uncertain.

Searches using the track channel using incoming muons entering the detector from below have now also confirmed this result in an almost independent channel. Starting from an analysis of two years of IceCube data which found a component beyond atmospheric background expectations of $3.7 \sigma$ [23] (see Fig. 2), 6 total years of IceCube data have now been analyzed and results will be published soon. Results from a 3-year sub-sample can be found later in these proceedings [24]. All these searches confirm the flux detected in the starting event channel. There are still large uncertainties on the spectral shapes and on flavor composition. A dedicated "global fit" to multiple IceCube analyses has been performed in order to use as much available information as possible to answer these questions [25]. Fig. 3 shows limits set on the flavor composition by this global fit. Some source flavor composition models such as neutron decay, where a pure flux of electron neutrinos is expected at the source, are already excluded by this analysis. 


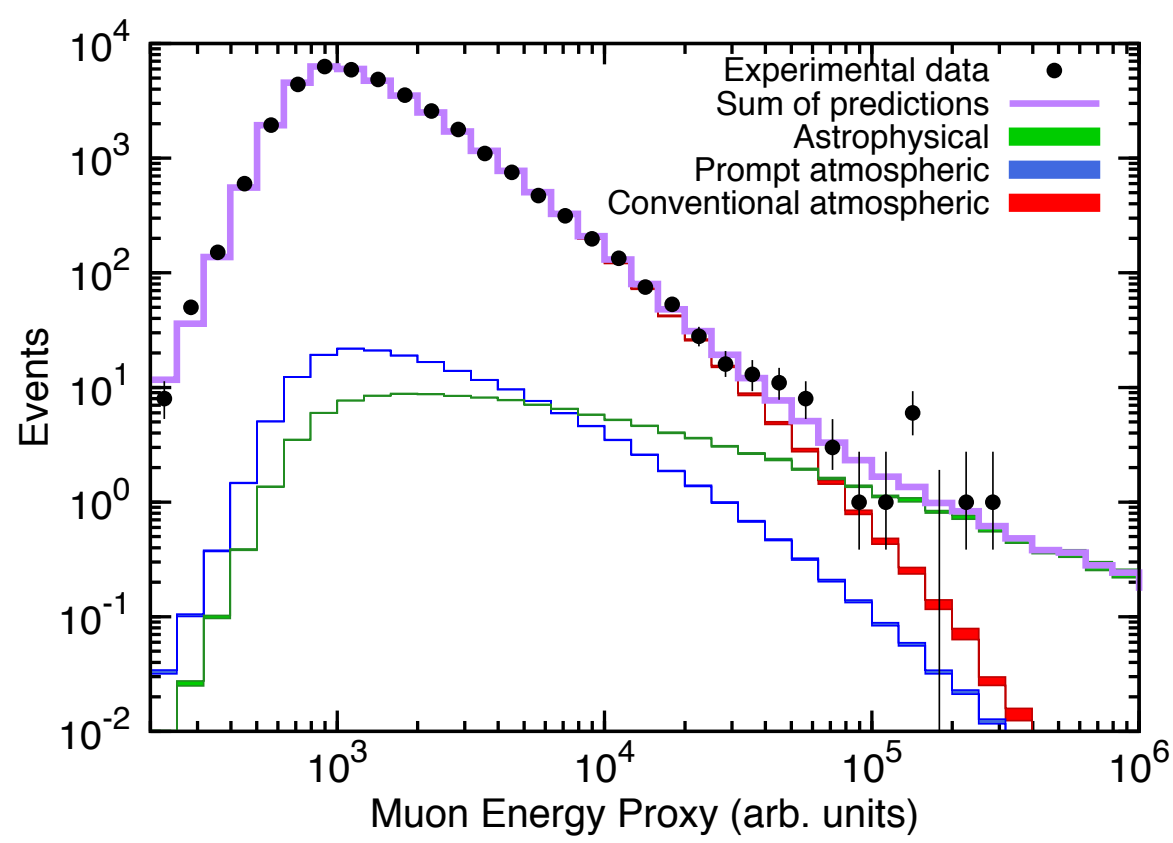

Figure 2: Distribution of a muon energy proxy variable of the final event sample used in a two-year trackbased study of the diffuse neutrino flux. Best-fit contributions of atmospheric backgrounds and an astrophysical component are shown as colored lines. An excess above atmospheric expectations can clearly be seen at the high-energy end of the distribution. [23]

\subsection{Point Source Searches}

The next step after detection of an astrophysical neutrino flux is to attribute it to sources or source classes. The more traditional way to do this is to use the "track" channel and search for clustering in the sky. These searches are dominated by atmospheric muons for down-going events and atmospheric neutrinos for up-going events. Both ANTARES and IceCube have performed such searches [26, 27], but neither has found significant clustering at any position on the sky. An overview of current limits on neutrino point sources from both ANTARES and IceCube can be seen in Fig. 4. Note that these point-source samples are dominated by atmospheric backgrounds in order to allow as much low-energy signal as possible to be accepted, which in turn maximizes the sensitivity to clustering (under the assumption that neutrino source spectra are steeply-falling power laws). In principle, searches for point sources in neutrinos should have their sensitivities scale proportional to the square root of observation time. Up to this point in time the scaling has been faster than that because of improvements in analysis technique (such as the inclusion of event energy) and reconstruction techniques (mostly improvements in reconstruction quality/angular error). IceCube has also performed a clustering study using the high-energy starting-event sample described earlier. Even though this sample should be signal-dominated, the limited amount of statistics and poor angular resolution of shower-type events limits the sensitivity of this search which has not yielded any significant clustering after trial-correction (see Fig. 5). Although not significant, the apparent clustering in Fig. 5 near the galactic center has been studied in detail by ANTARES. Their field of view for track-like events includes this region and allows them to extend searches for clustering to lower energies. None of these searches have found significant clustering. More recent point-source 


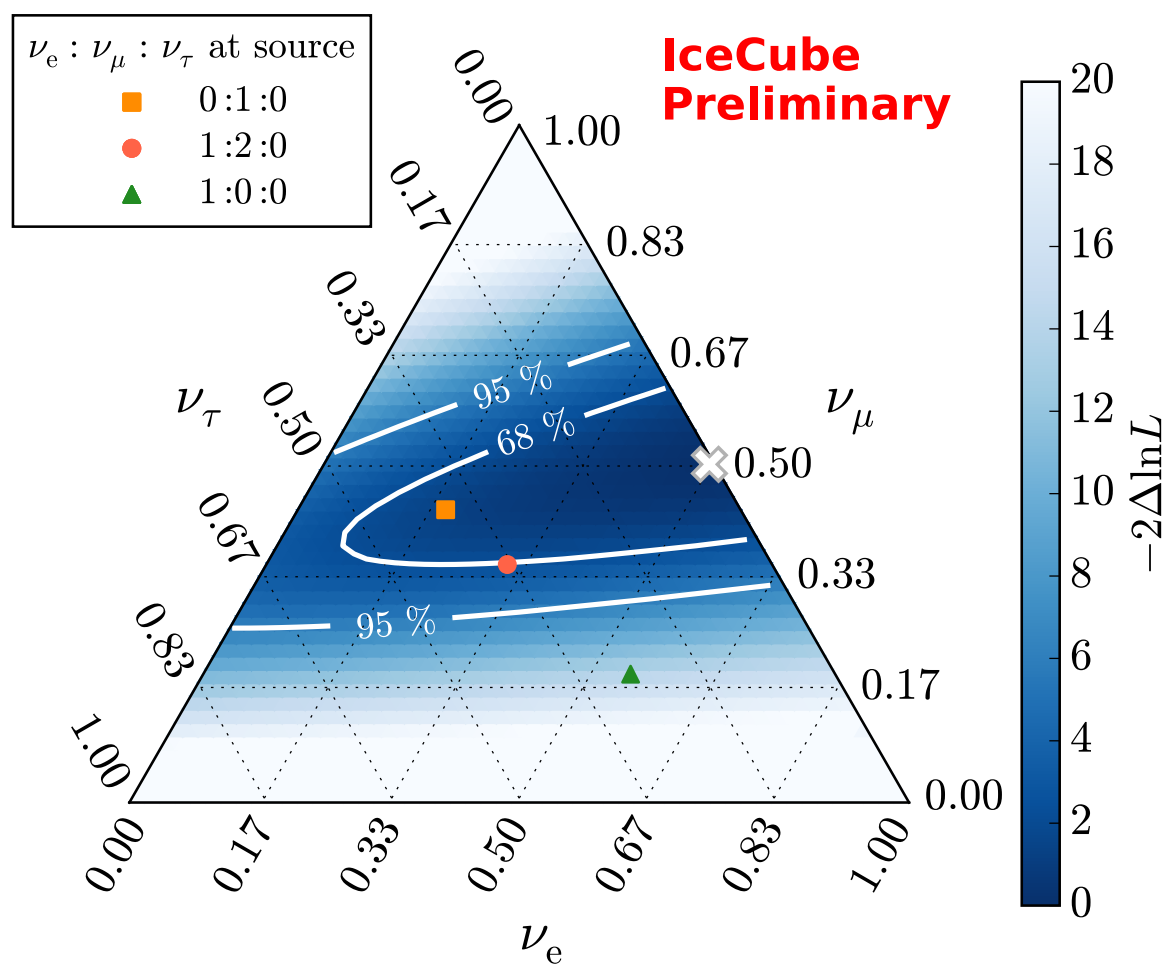

Figure 3: Constraints on flavor composition of the astrophysical neutrino flux from a global fit of various other IceCube analyses. Each point on the triangle corresponds to a ratio $v_{e}: v_{\mu}: v_{\tau}$ as measured at Earth. Compositions expected for three different source scenarios (before oscillations) are indicated. [25]

searches in IceCube and ANTARES are beginning to use the starting event channel in addition to the usual track channel to improve sensitivity to lower energies [28, 29]. Also point sources searches using a joint sample of IceCube and ANTARES data have been performed [30].

\subsection{Limits on Transient Sources}

One of the most prominent transient sources discussed as candidates for the production of astrophysical neutrinos are gamma-ray bursts (GRBs). These objects generate very bright $\gamma$-ray signals within a short duration of typically 1 to 100 seconds. Because of this short time span, the relevant background from atmospheric neutrinos to GRBs is significantly reduced and even single neutrino observations within a short time window coincident to a GRB would be of significant interest. No such coincidence has been reported so far, which places stringent limits on models of GRBs as the sources of cosmic rays of the reported neutrino flux [32]. It should be noted, however, that other transient phenomena such as off-axis or failed GRBs (GRBs where the jet does not escape the progenitor star) and several other models are not ruled out by this non-observation. To be sensitive to a wide range of transient phenomena, both ANTARES and IceCube search for event clusters in direction and time. Versions of these searches are run in real-time so that follow-up observations with other observatories are possible [33,34]. Triggers are (or were) sent to networks of small robotic telescopes (ROTSE and TAROT), the Palomar Transient Factory (PTF), the SWIFT X-ray 


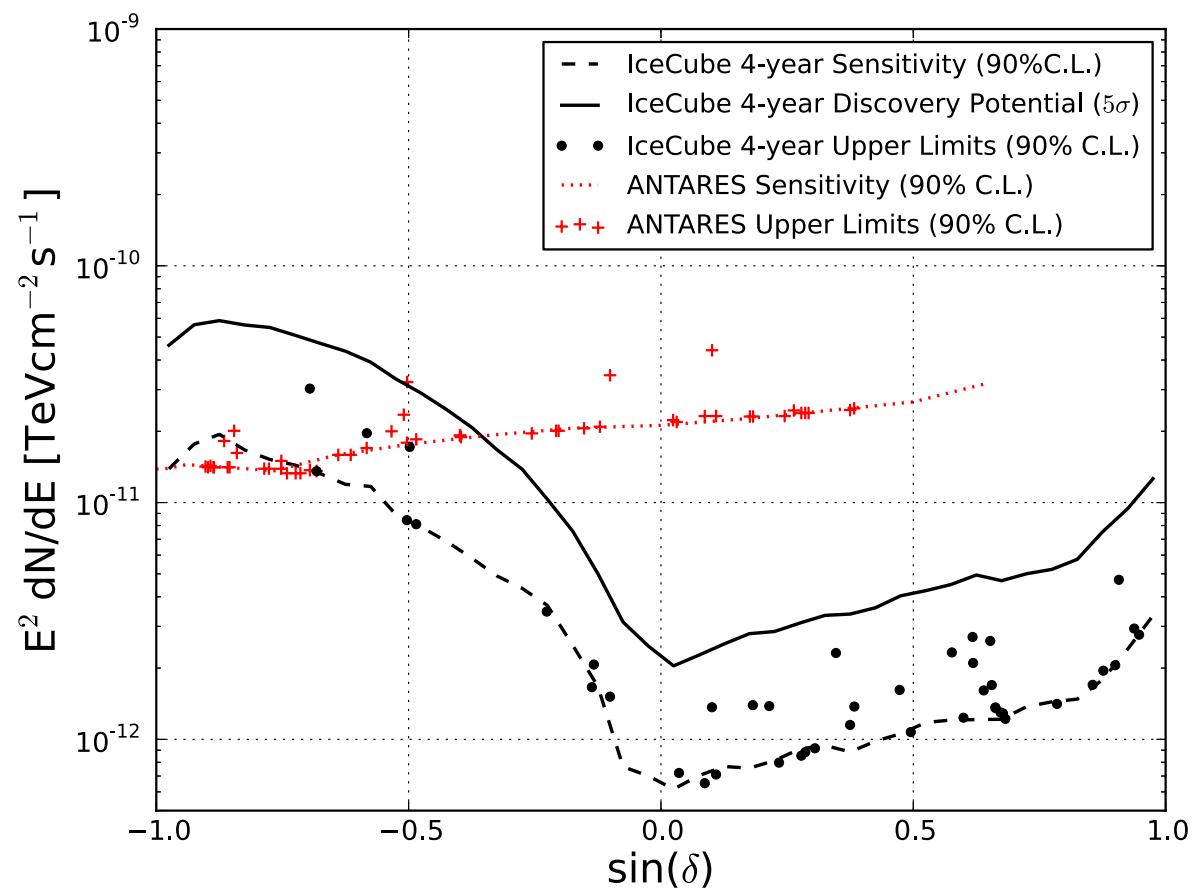

Figure 4: Sensitivity to an $E^{-2}$ neutrino flux from individual point sources as a function of declination [31]. The IceCube constraints are derived from the non-observation of clustering in data dominated by atmospheric muon neutrinos (Northern hemisphere) and atmospheric muons (Southern hemisphere), with correspondingly different energy thresholds. The constraints from ANTARES are derived from the nonobservation of clustering in a sample dominated by atmospheric neutrinos.

satellite and to IACTs such as MAGIC and VERITAS [35, 36]. IceCube and ANTARES alerts are also sent through the AMON network for distribution to various third-party experiments [37].

\subsection{GZK Neutrinos}

IceCube is also able to constrain models (or discover fluxes from) neutrinos generated by interaction of the highest-energy cosmic rays on photons of the cosmic microwave background. Such "cosmogenic" or "GZK" neutrino fluxes are characterized by broken power-laws above energies of a few PeV and extend up to several hundred EeV. Because IceCube has sensitivity to neutrinos of such energies, mainly due to its large volume, it is able to search for these fluxes. Studies of up to six years of recorded IceCube data are currently underway. Under favorable conditions (such as a proton composition of the primary cosmic rays), their sensitivity is expected to be sufficient for the first detection of GZK neutrinos in IceCube [38].

\subsection{Neutrino Physics and Dark Matter}

With the existence of dark matter firmly established by a number of independent observations, including anomalous rotation velocities of galaxies, gravitational lensing of distant galaxies, and anisotropy in the cosmic microwave background, the direct evidence of its particulate nature still remains elusive. Weakly Interacting Massive Particles (WIMPs), like the lightest stable supersymmetric (SUSY) particle (the neutralino), are particularly well motivated dark matter candidates and 


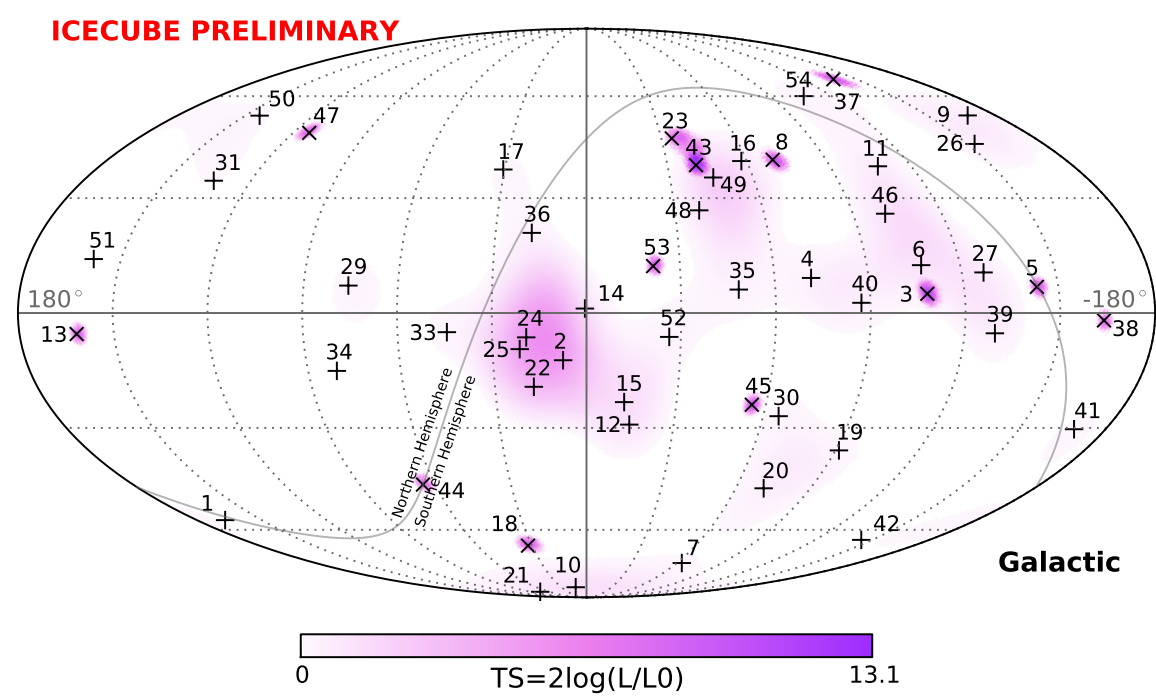

Figure 5: Arrival directions of the high-energy starting event sample in galactic coordinates. Shower-like events (with angular resolutions of the order of $10^{\circ}$ to $15^{\circ}$ ) are marked with + and those containing tracks (with resolutions better than $1^{\circ}$ ) with $\times$. Colors show the test statistics (TS) for the point-source clustering test at each location. No significant clustering was found. [22]

the focus of dozens of direct detection experiments worldwide. Given that the formation of largescale galactic structure is attributed to the distribution of dark matter, the largest local concentration is expected near the Milky Way galactic centre (GC) with predicted enrichment in massive objects, like the Sun, through gravitational capture. WIMPs that decay with very long lifetimes, or annihilate with each other, result in high energy Standard Model particles including neutrinos. Signals from these WIMP interactions and their measurements probe two fundamental properties: the WIMP-nucleon scattering cross section and either the self-annihilation cross section or lifetime. WIMP annihilation in the Sun, the GC and dwarf spheroidal galaxies would provide a striking signature given the expected energy spectrum of the events. Both IceCube and ANTARES are setting competitive limits on dark matter signals from neutrinos [39, 40].

While the focus in this article is mostly on astrophysical neutrino sources, it should be noted that the atmospheric neutrino flux measured by neutrino detectors can be used as a long-baseline neutrino source to study effects such as neutrino oscillations. IceCube's DeepCore array with its threshold of about $10 \mathrm{GeV}$ has made it possible to map out the oscillation minimum at $E_{v} \approx 25 \mathrm{GeV}$ in detail [41]. The current results are statistically limited and on-going analyses predict reaching limits as sensitive as other projects like T2K.

\section{Future Detectors}

Given the success of the IceCube detector and its DeepCore sub-array, a substantial detector upgrade to both lower and higher energies is currently being planned. The upgraded detector, named IceCube-Gen2, will consist of the low-energy extension, PINGU, and a high-energy upgrade. PINGU will consist of an even denser instrumentation of the region of deep glacial ice 
currently occupied by the DeepCore sub-array, lowering the energy threshold of the detector nearly an order of magnitude. This opens up the possibility to measure the neutrino mass hierarchy within a very competitive time-scale and for comparatively modest cost [42]. On the other end of the energy scale, a high-energy detector upgrade to IceCube will add more detector strings with optical modules around the current detector, significantly increasing the detector acceptance for the recently discovered high-energy astrophysical neutrino flux. This upgraded detector will have an improved effective area and angular resolution for the highest-energy events [43]. The high-energy array will permit a significant increase in the statistics of high-energy events, improved further by the addition of a full surface veto above the array.

In the Mediterranean, the KM3NeT project has recently started construction and deployed its first string of optical modules [16]. Its densely-spaced ORCA 'building block' will also be able to measure the neutrino mass hierarchy on a competitive time-scale while the ARCA high-energy array should be able to confirm the IceCube detection in a detector with different systematics and pointing resolution in order to help find the sources of the observed flux.

\section{Summary and Outlook}

Since the first evidence of astrophysical neutrinos in 2013, the significance of the observed signal has steadily increased with more and more observed data. Astrophysical neutrinos have now been observed in multiple channels (incoming tracks and starting events), allowing details such as their flavor composition to be mapped out. With increasing statistics the detailed properties of the flux are now being studied. While the distribution of the detected high-energy neutrinos in the sky is consistent with an extragalactic origin, a Galactic component can not be excluded at this time. The absence of a point source discovery has started to constrain the number of sources responsible for the flux - a single source responsible for the total flux would already have been discovered given the current point source sensitivities. The future KM3NeT/ARCA telescope which recently started construction should soon be able to provide an independent confirmation of IceCube's observation of the astrophysical neutrino flux. The sources of astrophysical neutrinos are not obvious at this time, but ongoing observations with current and future neutrino telescopes and the combination with information from radio, optical, X-rays and $\gamma$-rays should allow us to narrow down the possibilities and eventually lead to the discovery of the sources.

\section{References}

[1] M.G. Aartsen et al., Science 342, 1242856 (2013)

[2] R. Abbasi et al., PRL 111 (2013) 021103

[3] M. Ahlers, PoS(ICRC2015)022, these proceedings

[4] A. Ishihara, PoS(ICRC2015)013, these proceedings

[5] E. Waxman, 2013, Proceedings of Rencontres du Vietnam: Windows on the Universe (2013), arXiv:1312.0558.

[6] J. K. Becker, Phys. Rept. 458, 173 (2008)

[7] P. Desiati, PoS(ICRC2015)028, these proceedings 
[8] M. Honda, et al., PRD75 (2007) 043006

[9] R. Enberg, M. H. Reno, and I. Sarcevic, PRD 78 (2008) 043005

[10] M.G. Aartsen et al., JINST 9 (2014) P03009

[11] IceCube Coll., PoS(ICRC2015)1071, these proceedings

[12] S. SchÃúnert et al., PRD79 (2009) 043009

[13] T.K. Gaisser at al., PRD90 (2014) 023009

[14] G. Aggouras, et al. [NESTOR Collaboration], NIM A 552, 420 (2005)

[15] M. Taiuti et al. [NEMO Collaboration], NIM A 626, S25 (2011)

[16] C. James, PoS(ICRC2015)024, these proceedings

[17] B. Shaybonov, PoS(ICRC2015)1165, these proceedings

[18] R. Abbasi et al., NIM A 601, 294 (2009)

[19] IceCube Coll., PoS(ICRC2015)334, these proceedings

[20] M. G. Aartsen et al., PRD 89 (2014) 062007

[21] M. G. Aartsen et al., PRD 89 (2014) 102001

[22] IceCube Coll., PoS(ICRC2015)1081, these proceedings

[23] R. Abbasi et al., PRL 115 (2015) 081102

[24] IceCube Coll., PoS(ICRC2015)1079, these proceedings

[25] IceCube Coll., PoS(ICRC2015)1066, these proceedings

[26] T. Michael, PoS(ICRC2015)1078, these proceedings

[27] IceCube Coll., PoS(ICRC2015)1047, these proceedings

[28] IceCube Coll., PoS(ICRC2015)1056, these proceedings

[29] IceCube Coll., PoS(ICRC2015)1053, these proceedings

[30] ANTARES and IceCube Coll., PoS(ICRC2015)1076, these proceedings

[31] M. G. Aartsen et al., APJ 796 (2014) 109

[32] R. Abbasi et al., Nature 484351 (2012)

[33] R. Abbasi et al., Astron. Astrophys. 539, A60 (2012)

[34] M. Ageron et al., Astropart. Phys. 35, 530 (2012)

[35] IceCube Coll., PoS(ICRC2015)1052, these proceedings

[36] IceCube Coll., PoS(ICRC2015)1069, these proceedings

[37] G. Tesic, PoS(ICRC2015)329, these proceedings

[38] IceCube Coll., PoS(ICRC2015)1064, these proceedings

[39] IceCube Coll., PoS(ICRC2015)1209, these proceedings

[40] ANTARES Coll., PoS(ICRC2015)1207, these proceedings

[41] M. G. Aartsen et al., PRD91 (2015) 072004

[42] IceCube-Gen2 Coll., PoS(ICRC2015)1174, these proceedings

[43] IceCube-Gen2 Coll., PoS(ICRC2015)1146, these proceedings 